\title{
Stability of ABR congestion control using the theory of delayed differential equations
}

\author{
O. Ait-Hellal $\dagger^{*}$ and E. Altman $\ddagger \S$
}

\begin{abstract}
The stabilities of the Fixed Point, ERAQLES and ERICA+ABR congestion control algorithms were investigated. By using the stability theory of delayed differential equations, the stability conditions for each algorithm are obtained and examples where these algorithms are not stable (the stability definition is given below) are given. Parameters are proposed for which ERICA+ is always stable. It is shown that for a range of control parameters, neither the Fixed Point nor ERAQLES algorithms are stable, whatever the parameters are (round trip times, etc.).
\end{abstract}

\section{Introduction}

Adaptive mechanisms for congestion control have a central role in the efficient sharing of the network resources between many users. These mechanisms also have the role of preventing congestion in the network (Brakmo and Peterson 1995, Van Jacobson 1988). When control is performed by the sources (as is the case in the Internet) and not by the network, it makes it hard to protect the network from applications that might not use such mechanisms, e.g. from video conferences that use UDP (User Datagram Protocol, an Internet protocol that does not include any congestion control). In contrast, ATM networks (ATM Forum 1996) that offer guarantees on quality of service (delays, loss rates), need control (or police) mechanisms for which the network is responsible.This paper focuses on flow control problems arising in ATM. Note that guaranteeing quality of service could become a marginal control issue if available bandwidth becomes abundant. This would also marginalize the role of ATM networks. However, in view of the ongoing deployment of the third generation multimedia wireless networks, ATM becomes again central in networks (Holma and Toskala

Received 15 July 2002. Revised 25 December 2002. Accepted 1 June 2003.

$\dagger$ Department of Computer Science, Long Island University, 1 University Plaza, Brooklyn, NY 11201.

$\ddagger$ INRIA, BP 93, 2004 route des Lucioles, F-06902 Sophia-Antipolis Cedex, France.

$\S$ Present address: CESIMO, Facultad de Ingeneria, Universitad de Los Andes, Mérida, Venezuela.

*To whom correspondence should be addressed. e-mail: Omar.AitHellal@liu.edu
2001: Section 5.4.1): radio resources are scarce and network-controlled protocols are needed to prevent congestion.

In ATM networks, the Available Bit Rate (ABR) service (ATM Forum 1996) has been defined for supporting best-effort applications, in which the control decisions are taken by the network in the switches, ensuring that one achieves fairness among the active connections and controlling the loss cell ratios. This service manages the bandwidth left over by applications that have guaranteed performance: Variable Bit Rate (VBR) and Constant Bit Rate (CBR), and shares it between the ABR sources by signalling to them their allowable transmission rate. (In ATM Forum (1996), the ATM forum has standardized four transfer capacities (i.e. service types): the VBR and CBR, which are designed for real-time applications or for other services requiring some guarantees on delays and cell loss ratio, the ABR and Unspecified Bit Rate (UBR) which are best-effort service classes.) The behaviour of the source and destination is specified in ATM Forum (1996) as well as the manner in which feedback information should be conveyed back to the source (ATM Forum 1996, Jain et al. 1996). The behaviour of the switches, however, is left to the designer of the switch.

Several controllers have been proposed for the switches. They are either based on (1) the Explicit Forward Congestion Indication (EFCI) bit which originates from the approach of the DEC (Digital Equipment Corporation) bit (Ramakrishnan and Jain 1988); it indicates whether the congestion is detected or not, or (2) on Explicit Rate (ER), which informs the source on the bandwidth that is available (see 
Section 2 and (Chang et al. 1995, Charny et al. 1995, Jain et al. 1995, Jain 2003, Ohsaki et al. 1995b, Siu and Tzeng 1995), and references therein).

In the present paper, the stabilities of the widely known algorithms in each class are investigated. It is shown that for a range of parameters, there are always instabilities resulting in losses for both ERAQLES and Fixed Point algorithms. The stability domain is computed for the latter as well as for ERICA+ (Explicit Rate Indication for Congestion Avoidance) algorithm and examples of instability are given. In choosing the ABR algorithms to study, we did not attempt to be exhaustive or to study the algorithms with the best-known stability performance. Rather, we chose some important algorithms that seem to have had significance (in terms of implementations, standardization or discussions) in the ATM forum. (Examples of other algorithms known to be stable at steady-state are Altman et al. (1999), IEEE/ACM (1993), Chong et al. (2001), and Fulton et al. (1997)). (The importance of the algorithms is not necessarily in terms of their performance, but rather in the fact that they have been widely discussed in more industrial context. For example, ERICA+ is explicitly presented in the ATM specifications; ATM Forum 1996).

We should finally mention that there has been some related research on the stability of congestion control in networks with delayed information (Hollot et al. 2001, Johari and Tan 2001, Mossoulie 2003, Paganini et al. 2001, Shakkottai et al. 2001, Vinnicombe 2003) yet in the context of the the Internet, using also tools from delayed differential or difference equations.

The paper is structured as follows. Section 2 gives the mathematical tools used in the paper (stability definition and theorems related to the stability). EFCI queue length-based algorithms (Fixed Point) are presented in Section 3. In Section 4, ERAQLES and ERICA algorithms are presented and analysed. Finally, we conclude with some remarks and future work in Section 5.

\section{Background}

In what follows, we shall use the stability theory of delayed differential equations (also known as differential-difference equations). We are interested in this method, because an exact evaluation of such equations (differential equations with delay) is not known in many cases; hence only the stability can be analysed in some cases. This method is sufficient to discuss the stability of many rate based flow control algorithms (ERICA+ Jain et al. 1996; ERAQLES Moret and Fdida 1997; Fixed Point Kim et al. 1996), etc.).

We begin by stating the definition of the stability of a solution (Bellman and Cooke 1963) (local stability).
We will illustrate this for the following delayed differential equation

$$
\frac{\mathrm{d} x(t)}{\mathrm{d} t}=f(x(t), x(t-\tau), t) .
$$

Definition 2.1: Let $w(t)$ be a function, continuous for $t>0$, which satisfies the equation in (1) for $t>\tau$. This solution is said to be stable as $t \rightarrow \infty$ if, given two positive numbers $t_{0}$ and $\epsilon$, there exists a corresponding positive number $\delta$ such that every continuous solution $x(t)$ of the equation in (1) which satisfies

$$
\max _{t_{0} \leq t \leq t_{0}+\tau}|x(t)-w(t)| \leq \delta
$$

will also satisfy

$$
\max _{t_{0} \leq t}|x(t)-w(t)| \leq \epsilon .
$$

The solution is said to be uniformly stable if, given $\epsilon$, there exists a $\delta$ such that for any $t_{0}>0$ and any solution $x(t)$ which satisfies (2) will also satisfy (3).

The solution $w(t)$ is said to be asymptotically stable if

- it is stable; and

- for each $t_{0} \geq 0$ there is a $\delta$ such that every solution $x(t)$ which satisfies (2) will also satisfy

$$
\lim _{t \rightarrow \infty}[x(t)-w(t)]=0 .
$$

Theorem 2.1: Consider the following delayed differential equation

$$
\frac{\mathrm{d} x(t)}{\mathrm{d} t}+\alpha \frac{\mathrm{d} x(t-\tau)}{\mathrm{d} t}+\beta x(t)+\gamma x(t-\tau)=0 .
$$

Then the following statements are true:

- If $|\alpha|>1$, then the zero solution is unstable for all positive delay $\tau$.

- If $|\alpha|<1, \gamma^{2}<\beta^{2}$, or $\gamma=\beta \neq 0$, then increasing $\tau$ does not change the stability of the zero solution.

- If $|\alpha|<1, \gamma^{2}>\beta^{2}$, and

(i) $\gamma+\beta<0$, then the zero solution is unstable for all positive delay $\tau$; and

(ii) $\gamma+\beta>0$, then the zero solution is uniformly asymptotically stable when $\tau<\tau_{0}$ and unstable when $\tau>\tau_{0}$, where $\tau_{0}=\theta / w$, and

$$
\begin{aligned}
& w=\left(\left(\gamma^{2}-\beta^{2}\right)\left(1-\alpha^{2}\right)^{-1}\right)^{1 / 2}, \\
& \theta=\operatorname{arccot}\left(-\frac{\alpha w^{2}+\beta \gamma}{w(\gamma-\beta \alpha)}\right) .
\end{aligned}
$$


Next we state a generalization of the PoincaréLyapunov theorem (Bellman and Cooke 1963) to delayed differential equations.

Theorem 2.2: Consider the following nonlinear equation $a_{0} \frac{\mathrm{d} u(t)}{\mathrm{d} t}+b_{0} u(t)+b_{1} u(t-\tau)=f(u(t), u(t-\tau)), \quad t>\tau$,

where $a_{0} \neq 0, b_{0}, b_{1}$ are constants, with initial condition

$$
u(t)=g(t), \quad 0 \leq t \leq \tau .
$$

\section{Suppose that}

(a) every continuous solution of the linear part

$$
a_{0} \frac{\mathrm{d} u(t)}{\mathrm{d} t}+b_{0} u(t)+b_{1} u(t-\tau)=0
$$

approaches zero as $t \rightarrow \infty$;

(b) $f(u, v)$ is a continuous function of $u$ and $v$ in a neighbourhood of the origin $|u|+|v| \leq c_{1}$; and

(c)

$$
\lim _{|u|+|v| \rightarrow 0} \frac{|f(u, v)|}{|u|+|v|}=0 .
$$

Then, provided $\max _{0 \leq t \leq \tau}|g(t)|$ is sufficiently small (depending on $c_{1}, a_{0}, b_{0}$ and $b_{1}$ ), any solution of the nonlinear equation (5), can be continued over the interval $0 \leq t<\infty$, and each such solution satisfies

$$
\lim _{t \rightarrow \infty}|u(t)|=0
$$

The proofs of the Theorems can be found in Kuang (1993), respectively, Bellman and Cooke (1963) (Theorem 3.2, pp. 82 resp. Theorem 11.2 pp. 336).

Remark 2.1: We will encounter equations where $x(t)=0$ is a solution, which we call the 'zero-solution'. We shall analyze the stability of such a solution. If the zero solution is uniformly asymptotically stable, this means that all solutions converge to $x(t)=0$.

Remark 2.2: We shall ignore the delay between consecutive Resource Management (RM) cells, and consider that the Allowable Cell Rate (ACR), i.e. the rate at which the source is allowed to transmit is continuously updated. (It is through RM cells that the sources are informed of the congestion in the network. These cells are used as signalling mechanism to convey to the source information from the switches along the route from source destination, for updating the trasmission rate.)

\section{EFCI, queue length-based algorithms}

Several algorithms based on the congestion notification were proposed: PRCA, EPRCA, etc. (Brooillet and Madhow 1996, Ohsaki et al. 1995a,b, Siu and Tzeny 1995). Most of them translate the congestion to the buffer occupancy, so that the congestion is signalled depending on whether the buffer is larger or smaller than a given threshold. Theoretical approach in studying these schemes always considers a single bottleneck node.

In a recent study (Ait-Hellal and Altman 1999) where, mainly, the EFCI scheme was considered, it was shown that the maximum buffer size, necessary for avoiding overflow, strongly depended on the number of switches in the path when considering a single source; it may be even larger in case of multiple sources. This is because the notion of congestion differs from a switch to another if the switches have different thresholds, and the information about the congestion is binary (there is a congestion or there is not). Hence, continuous information (rather than a single bit information) on the buffer occupancy is quite desirable.

Indeed, other algorithms based on queue length have been proposed. The information conveyed by the switches back to the source are a function of the queue length at a time a backward RM cell is received. In Kim et al. (1996), a simple queue length-based scheme, called a Fixed Point algorithm, was presented. The scheme showed interesting features: convergence, and smoothness in the rate at the source. However, no extensive simulations or stability analysis were given. We present this algorithm as an example of the queuelength-based algorithms and analyse its stability. It is shown that for a range of parameters, the scheme is not stable whatever the round trip time.

\subsection{Fixed Point algorithm}

This algorithm is essentially based on the queue size at the intermediate switches. More precisely, it is based on the degree of saturation of each switch (i.e. the fraction of the queue size over a certain threshold, which is some given parameter, and which may be the maximum buffer size at the switch). The aim is to keep the queue length close to the threshold by varying the rate at which the source sends its data, inversely proportional to the buffer occupancy.

It is obvious that this algorithm is better than those based on the linear increase exponential decrease of the rate, namely EFCI-based switch algorithms in all 
performance criteria. It has the same complexity while it requires fewer buffers and allows different sources to get relatively the same bandwidth (fairness is achieved in the long run).

In this algorithm, RM cells are sent following the recommendation of ATM Forum (1996). The source is allowed to send data at a rate not greater than $\mathrm{PCR}_{i}$ (PCR stands for Peak Cell Rate). (The use of several letters to donote a single variable is standard in industrial community and in particular in the ATM Forum (1996).) At the beginning the source $i$ sends its data at rate $\mathrm{ICR}_{i}$ (ICR stands for Initial Cell Rate). In our analysis we do not take into account the initial conditions (note that even if the system is uniformly asymptotically stable, stability is not guaranteed for all initial conditions!). The rate at the source may increase or decrease according to the information carried in the backward RM cells $(y(t) / Q)$. Each time an RM cell is received at the source, the source adjusts its allowable cell rate ACR as follows:

$$
\mathrm{ACR}:=\mathrm{ACR}+\mathrm{AIR} * N_{r m}\left(1-\frac{\mathrm{ACR}}{\mathrm{PCR}}\right)-\mathrm{TQ} * \mathrm{ACR},
$$

where $N_{r m}$ is some constant (defined as the maximum number of cells a source may send for each forward RM cell. (According to ATM Forum (1996) in an ABR connection, the source is responsible for creating RM cells (called forward) and sending them (along with the rest of its packets) to the destination. When they reach the destination, the RM cells are sent back to the source after being marked by congested nodes to signal to the source how to update its transmission rate.) AIR is defined as the Additive Increase Rate (ATM Forum 1996); in the absence of congestion, it defines the allowable increase in the transmission rate. TQ is a field in the coming RM cell; when a backward $\mathrm{RM}$ cell is received at the switch, the field TQ is set to the queue size at the switch divided by the maximum queue size (buffer capacity).

$$
\mathrm{TQ}:=\frac{\text { queue size }}{\text { maximum queue size }} \text {. }
$$

\subsection{Analytic approach}

Here we aim to analyse this algorithm and find out its insufficiencies. We use the delayed differential equations to model it. We consider several equal saturated sources sending to the same sink and sharing a bottleneck node whose capacity is $\mu$, as constant. The round trip time for an RM cell is $\tau$. The buffer size is of $Q$ buffers.
Define:

$\beta_{i} \triangleq$ time it takes a cell from the bottleneck (queue) to reach the source $i$,

$\gamma_{i} \triangleq$ time it takes a cell from source $i$ to reach the bottleneck (queue),

$\tau_{i} \triangleq \beta_{i}+\gamma_{i}$,

$y(t) \triangleq$ queue size at time $t$,

$x(t) \triangleq$ rate of the source at time $t$,

$\bar{y} \triangleq$ queue size when the system stabilizes.

We suppose that the queue never empties and fairness is guaranteed among the $N$ sources. (The mode in which the queue never empties is the one corresponding to the desirable operation of the flow control, since it guarantees full utilization; the assumption on fairness among the sources is required by the ATM Forum (1996). We shall thus make these two assumptions in the sequel.) From equations (10) and (11), we have the following:

$$
\left\{\begin{array}{rl}
x_{i}(t)= & x_{i}\left(t^{-}\right)+\mathrm{AIR} * N_{r m}\left(1-\frac{x_{i}\left(t^{-}\right)}{\mathrm{PCR}}\right) \\
& -\frac{y\left(t-\beta_{i}\right)}{Q} x_{i}\left(t^{-}\right) \\
\frac{\mathrm{d} y(t)}{\mathrm{d} t}= & \sum_{i=1}^{N} x_{i}\left(t-\gamma_{i}\right)-\mu
\end{array},\right.
$$

where $t^{-}$is the last time the ACR was updated (the last time an RM cell has arrived). In the above delayed differential equations, each variable depends on the delayed value of the other one. We shall use some heuristic to simplify the first equation, which would allow us to study a one-dimensional delayed differential equation instead. Although this would only be an approximation that might not always be valid, it will allow us to get a simple characterization of the stability region. This would then help us find, when using simulations, a more accurate characterization of the stability region.

Our approach is based on replacing the first equation by a fixed-point approximation, setting $x(t)=x\left(t^{-}\right)$. The approximation can be justified and understood, for example, if we assume that $y(t)$ varies sufficiently slow with respect to $x(t)$ so that in the second equation in (12). It is then related to the boundary layer approach for differential equations which have two time scales (e.g. Khalil 1996, Kanniyur and Srikant 2001). The latter is based on first studying the behaviour of the fast changing variable assuming that the slow variable is frozen. This amounts on focusing on the first equation in (12), and taking $y$ there to be a constant. It is then easily seen that $x(t)$ is asymptotically stable under this assumption and converges to some limiting value. (In spite of the above motivation, we do not attempt 
to provide a rigorous justification for our approximation. Our approach is rather to use it as a heuristic in order to help us in guessing the stability region, which is then obtained more precisely using simulations.) Therefore, for a fixed $y$ in that equation, we approximate $x$ by its limiting value (which is a function of that $y(t)$, and is denoted by $x(t)$ with some abuse of notation). We then substitute this value in the second equation in (12) and obtain

$$
\left\{\begin{array}{rl}
x_{i}(t) \cong & \mathrm{AIR} * N_{r m} * \\
& \left(\frac{\mathrm{PCR} * Q}{\mathrm{AIR} * N_{r m} * Q+\mathrm{PCR} * y\left(t-\beta_{i}\right)}\right) \\
\frac{\mathrm{d} y(t)}{\mathrm{d} t}= & \sum_{i=1}^{N} x_{i}\left(t-\gamma_{i}\right)-\mu
\end{array} .\right.
$$

Substituting in the first equation of $t-\gamma_{i}$ instead of $t$, multiplying both sides by the denominator, and summing over $i$, we obtain

$$
\begin{aligned}
\sum_{i=1}^{N} \operatorname{AIR} * N_{r m}= & \frac{\operatorname{AIR} * N_{r m}}{\operatorname{PCR}} \sum_{i=1}^{N} x_{i}\left(t-\gamma_{i}\right) \\
& +\frac{1}{Q} \sum_{i=1}^{N}\left(y\left(t-\tau_{i}\right) x_{i}\left(t-\gamma_{i}\right)\right) .
\end{aligned}
$$

Hence,

$$
\begin{aligned}
\frac{\mathrm{d} y(t)}{\mathrm{d} t}= & \sum_{i=1}^{N} x_{i}\left(t-\gamma_{i}\right)-\mu \\
= & \sum_{i=1}^{N} x_{i}\left(t-\gamma_{i}\right)+\sum_{i=1}^{N} \mathrm{AIR} * N_{r m} \\
& -\frac{\operatorname{AIR} * N_{r m}}{\operatorname{PCR}} \sum_{i=1}^{N} x_{i}\left(t-\gamma_{i}\right) \\
& -\frac{1}{Q} \sum_{i=1}^{N}\left(y\left(t-\tau_{i}\right) x_{i}\left(t-\gamma_{i}\right)\right)-\mu \\
= & \left(1-\frac{\operatorname{AIR} * N_{r m}}{\operatorname{PCR}}\right) \frac{\mathrm{d} y(t)}{\mathrm{d} t} \\
& +\frac{(N * \operatorname{PCR}-\mu) \operatorname{AIR} * N_{r m}}{\operatorname{PCR}} \\
& -\frac{1}{Q} \sum_{i=1}^{N}\left(y\left(t-\tau_{i}\right)\left(x_{i}\left(t-\gamma_{i}\right)-\frac{\mu}{N}+\frac{\mu}{N}\right)\right) .
\end{aligned}
$$

Let

$$
H(t) \triangleq y(t)-\frac{(N * \mathrm{PCR}-\mu) \mathrm{AIR} * N_{r m} * Q}{\mu * \mathrm{PCR}}
$$

and

$$
\frac{\mathrm{d} H_{i}(t)}{\mathrm{d} t} \triangleq x_{i}\left(t-\gamma_{i}\right)-\frac{\mu}{N} \Longrightarrow \sum_{i=1}^{N} \frac{\mathrm{d} H_{i}(t)}{\mathrm{d} t}=\frac{\mathrm{d} H(t)}{\mathrm{d} t}
$$

Then from (14), we have

$$
\begin{aligned}
& \frac{\mathrm{d} H(t)}{\mathrm{d} t}+\frac{\mu^{2}}{N^{2} * \operatorname{AIR} * N_{r m} * Q} \sum_{i=1}^{N} H\left(t-\tau_{i}\right) \\
& =-\frac{\mu}{N * Q * \mathrm{AIR} * N_{r m}} \sum_{i=1}^{N} H\left(t-\tau_{i}\right) \frac{\mathrm{d} H_{i}(t)}{\mathrm{d} t} .
\end{aligned}
$$

A sufficient condition for uniform asymptotic stability is now presented.

Proposition 3.1: $\quad$ For homogeneous sources $\left(\tau_{i}=\tau \forall i\right)$, if

$$
\tau<\tau_{0} \triangleq \frac{\pi}{2} \frac{N * Q * \mathrm{AIR} * N_{r m}}{\mu^{2}}
$$

then the zero-solution of (15)

$$
\left(H(t)=0 \Longleftrightarrow y(t)=\frac{(N * \mathrm{PCR}-\mu) \mathrm{AIR} * N_{r m} * Q}{\mu * \mathrm{PCR}}\right)
$$

is uniformly asymptotically stable.

Proof: The proof follows from Theorems 2.1 and 2.2 after remarking that for homogeneous sources, we have

$$
\begin{aligned}
(15) \Longleftrightarrow & \frac{\mathrm{d} H(t)}{\mathrm{d} t}=-\frac{\mu^{2}}{N * \mathrm{AIR} * N_{r m} * Q} H(t-\tau) \\
& *\left(\frac{N * \mathrm{AIR} * N_{r m} * Q}{N * \mathrm{AIR} * N_{r m} * Q+\mu H(t-\tau)}\right) \\
\Longleftrightarrow & \frac{\mathrm{d} H(t)}{\mathrm{d} t}+\frac{\mu^{2}}{N * \mathrm{AIR} * N_{r m} * Q} H(t-\tau) \\
= & \frac{1}{N * \operatorname{AIR} * N_{r m} * Q} \\
& \times \frac{\mu^{3}(H(t-\tau))^{2}}{N * \operatorname{AIR} * N_{r m} * Q+\mu H(t-\tau)} \\
\triangleq & f(H(t-\tau)) .
\end{aligned}
$$

From Theorem 2.1, if $\tau \leq \tau_{0}$, then the zero-solution of the linear part of equation (16) is asymptotically stable. Hence, every solution of the linear part of equation (16) approaches zero as $t$ goes to $\infty$. This means that condition (a) of Theorem 2.2 holds. We note that

$$
\lim _{|u| \rightarrow 0} \frac{|f(u)|}{|u|}=0,
$$


so that condition (c) of Theorem 2.2 holds. Condition (b) clearly holds as well. We can thus apply Theorem 2.2 to equation (16), and conclude that the zero solution of that equation is asymptotically stable.

Figure 1 shows that the turning point between the stability and instability regions of the Fixed Point algorithm is very close to $\tau_{0}\left(\tau>\tau_{0}\right)$. The figure was obtained using simulations of the exact dynamics of the flow control mechanism (implemented on the REAL simulator) with the following parameters. We considered one source going through a single switch where the available bandwidth is $\mu=5000 \mathrm{cells} / \mathrm{s}$ $(1.272 \mathrm{Mbp}) . \quad \mathrm{AIR}=128, \quad \mathrm{PCR}=33333.3250 \mathrm{cells} / \mathrm{s}=$ $14.13 \mathrm{Mbp} \quad Q=200$ and $\mathrm{ICR}=2000 \mathrm{cells} / \mathrm{s}$. From Proposition 3.1, we get $\tau_{0}=0.05147 \mathrm{~s}$. Thus, the trivial solution of (16) is stable for $\tau \leq \tau_{0}$. Figure 1 shows that for the Fixed Point algorithm, the system is not stable for that value $(\tau=0.050 \mathrm{~s})$. However, it is stable for values, lightly, smaller $\left(\tau=0.044 \mathrm{~s}>\tau_{0}-1 /\right.$ AIR $)$.

In fact the value obtained in Proposition 3.1 is optimistic (since it predicted stability for $\tau<\tau_{0}$, whereas we see that at $\tau=0.05<\tau_{0}$ the system is still unstable). Yet the unstable region for the Fixed Point algorithm is, indeed, very close to that value (since we see that for $\tau=0.044$ it is already stable). The small error in predicting the stability region is probably due to the approximation used in deriving (13). The following heuristic is generally true: for the Fixed Point algorithm, the system is stable for $\tau<\tau_{1}$ and unstable for $\tau>\tau_{1}$ where $\tau_{1}$ satisfies $\tau_{0}>\tau_{1}>\tau_{0}-1 /$ AIR.

Now suppose that the system is stable (the rate at the sources stabilizes as well as the queue length), then (after setting $Q=\xi B, \xi<1$ ), from Proposition 3.1 we get

$$
\begin{aligned}
\bar{y} & =\frac{(N * \mathrm{PCR}-\mu) \mathrm{AIR} * N_{r m} * Q}{\mu * \mathrm{PCR}} \\
& =\frac{(N * \mathrm{PCR}-\mu) \mathrm{AIR} * N_{r m} * \xi B}{\mu * \mathrm{PCR}} .
\end{aligned}
$$

This result can be found in Kim et al. (1996). Note that the buffer size when the system stabilizes can exceed the maximum buffer size for some values of $\mu$.

Remark 3.1: The switch may experience losses (there is no stability), if

$$
\mu<\frac{N * \mathrm{PCR}}{1+\mathrm{PCR} /\left(\mathrm{AIR} * N_{r m} * \xi\right)}
$$

in which case the sufficient stability condition of Proposition 3.1 does not hold (setting $\bar{y}>B$ ). The limitation due to the smaller available bandwidth constitutes the main inconvenience for the proposed (a)

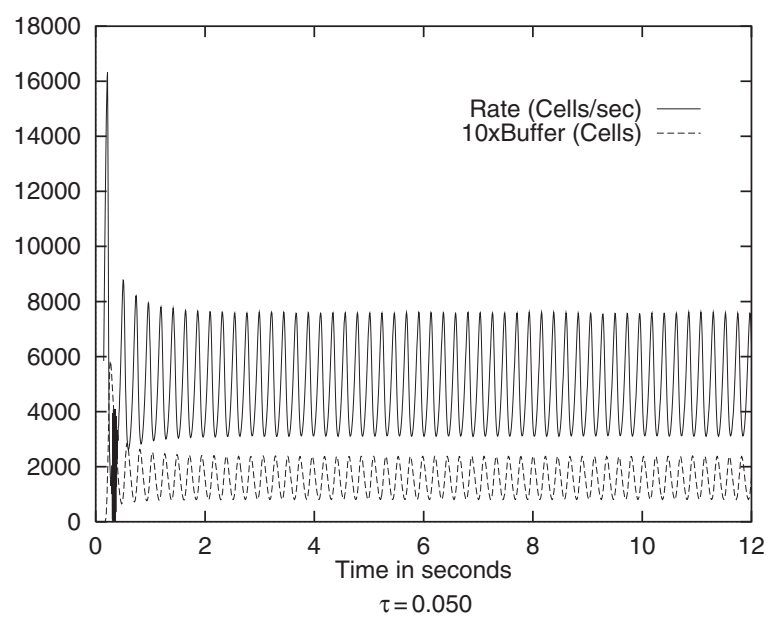

(b)

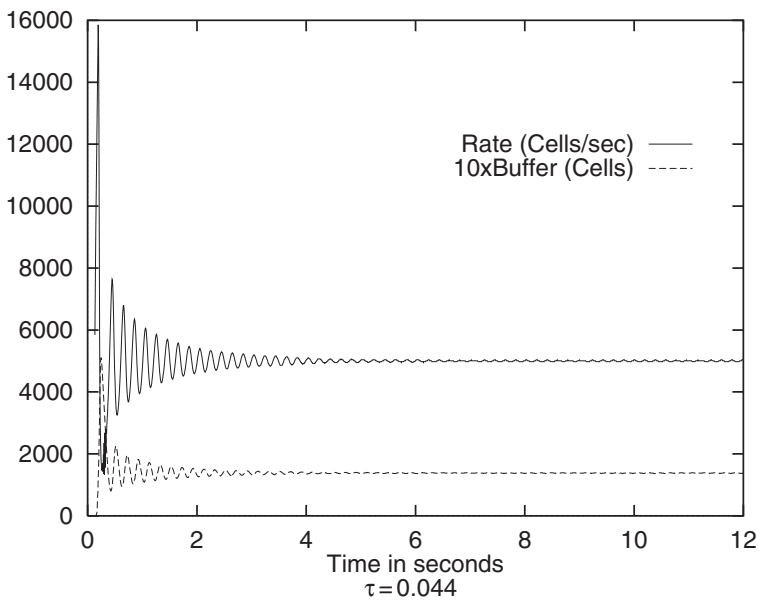

Figure 1. Buffer occupancy (cells) and the rate (cells/s), for $\tau=0.044 \mathrm{~s}$ (stable) and $\tau=0.050 \mathrm{~s}$ (non-stable), for the fixed point algorithm.

algorithm, even if a fraction of the maximum queue size (a certain threshold) is considered rather than the maximum queue size itself. On the other hand, large available bandwidth may cause instability.

\section{ER-based algorithms}

\subsection{ERAQLES algorithm}

The ERAQLES algorithm (Moret and Fdida 1997) (Explicit Rate Algorithm using Queue Length States) proposes to evaluate the leftover CBR VBR bandwidth in an another manner than ERICA, by using the variation of ABR queue length. In the sequel, we suppose that the available bandwidth is well estimated (as in the ERICA algorithm). This algorithm is close to the ERICA algorithm, since the attributed rate to each source is proportional to its ratio in the total incoming ABR traffic $\mathrm{CCR}_{i} / \mathrm{CCR}$ (utilization of the Load_ Factor 
in ERICA; see the next section for the ERICA description). Here $\mathrm{CCR}_{i}(t)$ is the rate of source $i$ at time $t$ and $\operatorname{CCR}(t)$ is the sum of the rates of all sources. It can be summarized, briefly, as follows (at the source, the rate is updated according to the ER field in incoming RM cells to the source):

\subsubsection{At the switch}

The explicit rate for source $i$ is computed using

$$
\mathrm{ER}_{i}(t)=\mathrm{ER}(t) \cdot s_{i}(t),
$$

where $s_{i}(t)$ is the fair share function, it is computed using

$$
s_{i}(t)=\left(1-s_{0}\right) \frac{\mathrm{CCR}_{i}(t-T)}{\operatorname{CCR}(t-T)}+s_{0}
$$

$0<s_{0}<1$ and $T$ is the time between the source and the switch.

$\operatorname{ER}(t)$ is the bandwidth not used by other higher priority (CBR, VBR) traffics plus the bandwidth able to fill up the buffer capacity during a feedback $(\tau)$ :

$$
\operatorname{ER}(t)=C^{*}+h \frac{Q-y(t)}{T},
$$

where $C^{*}$ is the leftover CBR VBR traffic, and $y(t)$ is the queue length at time $t$. $Q$ is a given threshold $(Q=\xi B$, $\xi<1$ and $B$ is the maximum buffer size). $h$ is an important parameter in ERAQLES (Moret and Fdida 1997: equation (2)). The larger it is, the slower the convergence is (Moret and Fdida 1997: Section 3.2). Note that in the case of low ABR buffer utilization, the sources are allowed to exceed the capacity $C^{*}$ by a value equal to $h Q / T$ (Moret and Fdida 1997: Section 3.2).

\subsubsection{Analytic approach}

Consider the same model and parameters as previously, then the evolution of $x(t)$ and $y(t)$ is given by:

$$
\left\{\begin{array}{c}
x_{i}(t)=\left(\left(1-s_{0}\right) \frac{x_{i}\left(t-\tau_{i}\right)}{\sum_{\ell=1}^{N} x_{\ell}\left(t-\gamma_{\ell}-\beta_{i}\right)}+s_{0}\right) \\
\times\left(\mu+h \frac{Q-y\left(t-\beta_{i}\right)}{T}\right) \\
y(t+\delta t)=\left(y(t)+\left(\sum_{i=1}^{N} x_{i}\left(t-\gamma_{i}\right)-\mu\right) \delta t\right)^{+}
\end{array}\right.
$$

Let

$$
H(t) \triangleq y(t)-Q-\frac{(N-1) s_{0} \mu T}{\left((N-1) s_{0}+1\right) h} .
$$

After some calculations, considering that the buffer never empties, taking homogeneous sources, and by following the same steps as previously, we get

$$
\frac{\mathrm{d} H(t)}{\mathrm{d} t}+h \frac{(N-1) s_{0}+1}{T} H(t-\tau)=0 .
$$

From Theorem 2.1, the zero solution of (21)

$$
\left(H(t)=0 \Longleftrightarrow y(t)=Q+\frac{(N-1) s_{0} \mu T}{\left((N-1) s_{0}+1\right) h}\right)
$$

is uniformly asymptotically stable if and only if

$$
\tau<\tau_{0}=\frac{\pi T}{2 h\left((N-1) s_{0}+1\right)} .
$$

Hence, the scheme is not stable for all parameters, its stability depends, mainly, on $h$ and $s_{0}$. If $h$ satisfies $\left(s_{0}=1 / N, 2 T=\tau\right)$

$\frac{\pi}{4 h\left((N-1) s_{0}+1\right)}<1 \Longrightarrow \frac{\pi}{4\left((N-1) s_{0}+1\right)} \leq 0.785 \leq h$,

then no stability is guaranteed. However, the parameters recommended in Moret and Fdida (1997) $\left(s_{0}=1 / N\right.$ and $h=0.1839)$ ensure the stability in all cases. Figure 2 illustrates the case of one source for $h=0.785$ (unstable) and 0.70 (stable). The parameters considered are the same parameters as previous (Fixed Point algorithm, unstable case $T=0.05 \mathrm{~s}$ ).

As we can see from equation (20), when the system stabilizes, the queue length depends on the available bandwidth, as is the case for the Fixed Point algorithm. After some calculations (we consider that $Q=\xi B$ where $B$ is the maximum buffer size; $\xi<1$ ), in the same manner as for the Fixed Point algorithm we prove the following lemma (using equation (22)).

Lemma 4.1: The switch experiences losses (there is no stability) for

$$
\frac{\tau}{2} \geq \frac{(1-\xi) B\left((N-1) s_{0}+1\right) h}{(N-1) s_{0} \mu} \Longrightarrow \tau \geq \frac{\pi}{2} \frac{(1-\xi) B}{(N-1) s_{0} \mu}
$$

We can consider $s_{0}=0$ to get a stable system in all cases. However, the proof of the fairness Moret and Fdida (1997) (even the proof was stated, only for equidistant sources) will not be valid. In fact for $s_{0}$ being too small, fairness is achieved in a relatively long time, and for $s_{0}$ being large, the stability might be non-guaranteed; additionally the queue length might exceed the maxi- 
(a)

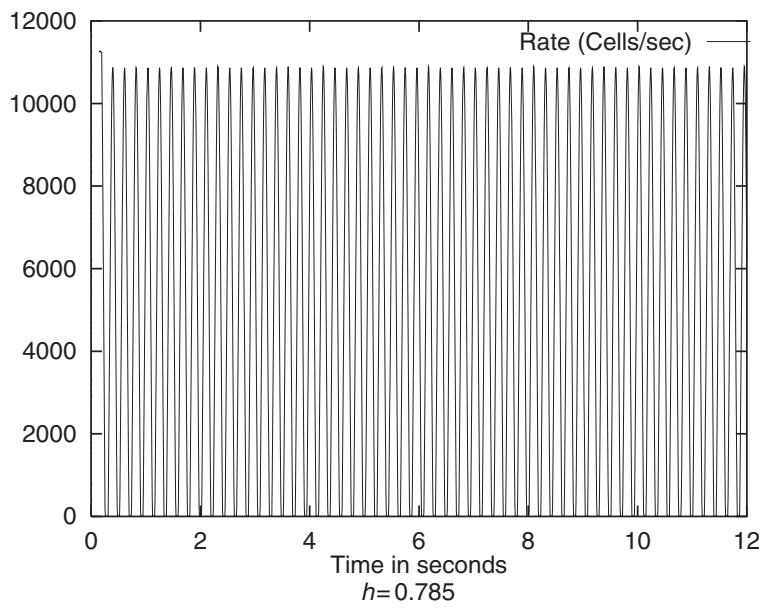

(b)

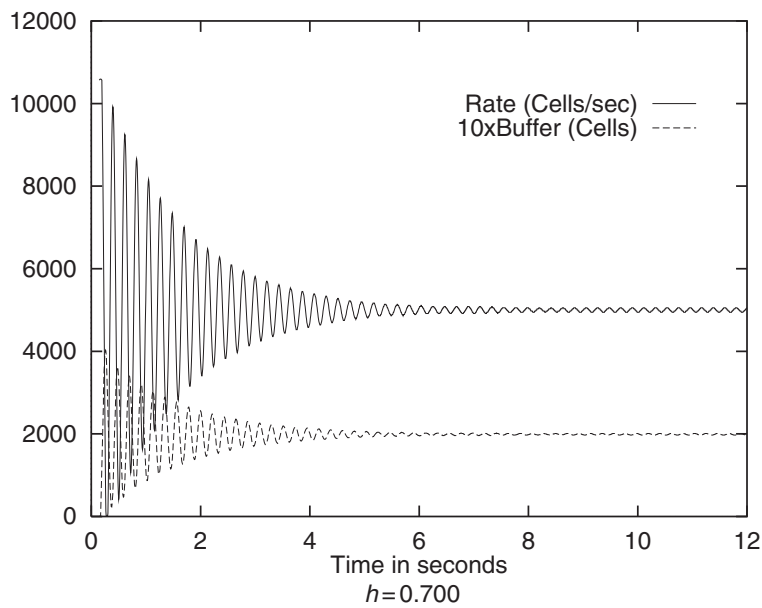

Figure 2. Rate in cells/s for $h=0.70$ and 0.785 , for the ERAQLES algorithm.

mum buffer size (see Lemma 4.1). This is the main disadvantage of this algorithm.

\subsection{ERICA(+) switch algorithm}

ERICA+, proposed by Jain et al. (1996), is an algorithm based on the available bandwidth together with the buffer occupancy at intermediate switches. It is the most referenced switch algorithm. Extensive simulations were done and many enhancements proposed. In all simulations, the scheme is reported as being stable. The present section aims to investigate the stability of the scheme by considering different parameters.

The ERICA switch algorithm works as follows. (For the lack of space many parameters were neglected, and only a sketch of the algorithm is presented in this paper. For more details see Jain et al. (1996).)

The switch determined periodically on each link is the load factor, $z$, the available capacity and the number $(N)$ of active Virtual Connections (VCs). The load factor is computed as:

$$
z \leftarrow \frac{\text { ABR input rate }}{\text { ABR capacity }}
$$

where

$$
\begin{gathered}
\text { ABR capacity } \leftarrow \text { target utilization } \times \text { link bandwidth } \\
- \text { VBR usage }- \text { CBR usage. }
\end{gathered}
$$

In order to use $100 \%$ of the available bandwidth, ERICA introduces the buffer occupancy as a control parameter (ERICA+) and Target Utilization is set to 1 , so that ABR capacity is revised as (we consider a constant threshold $(Q)$ ):

ABR capacity $\leftarrow$ ABR capacity $\times f n$ (Queue length),

where

$$
f n(\text { Queue length }) \triangleq \frac{b * Q}{(b-1) \times \text { queue length }+Q},
$$

where the recommended values for $b$ are

$$
b=1.15 \text { if queue length }>Q ; \quad b=1.05 \text { otherwise }
$$

Finally the rate for each connection is updated as follows (recall that the load factor also is changed):

$$
\begin{gathered}
\text { ER_calculated } \leftarrow \\
\max \left(\frac{\mathrm{ABR} \text { capacity }}{\text { number of active sources }}, \frac{\mathrm{CCR}[\mathrm{VC}]}{z}\right),
\end{gathered}
$$

$\mathrm{CCR}[\mathrm{VC}]$ is the rate in the incoming forward RM cell of the $\mathrm{VC}$, if the per $\mathrm{VC}$ counting is not supported; otherwise it is the input rate of that $\mathrm{VC}$ (number of input cells/averaging interval).

\subsubsection{Analytic approach}

To analyse the stability of ERICA+, we considered the case where ERICA aims a $100 \%$ utilization, and we used a constant queue threshold $(Q)$. The rate and the buffer size update in ERICA+ can be summarized in the following equations (we neglect the delay between RM cells).

Consider the same model and parameters as previously:

$$
\left\{\begin{array}{rl}
x_{i}(t)= & \max \left(\frac{1}{N}, \frac{x_{i}\left(t-\tau_{i}\right)}{\sum_{\ell=1}^{N} x_{\ell}\left(t-\gamma_{\ell}-\beta_{i}\right)}\right) \\
\times & \frac{\mu b Q}{(b-1) y\left(t-\beta_{i}\right)+Q} \\
y(t+\delta t)=\left(y(t)+\left(\sum_{i=1}^{N} x_{i}\left(t-\gamma_{i}\right)-\mu\right) \delta t\right)^{+}
\end{array},\right.
$$


where $b$ takes two values, as discussed above, depending on whether the queue length is larger or smaller than $Q$ (the recommended values are 1.15 respectively 1.05 ). In the analysis, we consider the value as being unique and we will identify the stability domain in the worst case (which will correspond to the smallest value of $b$ ).

Let

$$
H(t) \triangleq y(t)-Q
$$

Proceeding in the same manner as in the above section, after some calculations for the case of homogeneous sources, we get from (23)

$$
\frac{\mathrm{d} H(t)}{\mathrm{d} t}+\frac{(b-1) \mu}{b Q} H(t-\tau)=-\frac{(b-1)}{b Q} H(t-\tau) \frac{\mathrm{d} H(t)}{\mathrm{d} t} .
$$

\section{Proposition 4.1: If}

$$
\tau<\tau_{0}=\frac{\pi}{2} \frac{b Q}{(b-1) \mu}
$$

then the zero solution of $(24)(H(t)=0 \Longleftrightarrow y(t)=Q)$ is uniformly asymptotically stable.

Proof: The proof is similar to that of Proposition 3.1, after remarking that

$$
\begin{aligned}
&(24) \Longleftrightarrow \frac{\mathrm{d} H(t)}{\mathrm{d} t}= \\
& \quad-\frac{(b-1) \mu}{b Q} H(t-\tau) \\
& \quad \times\left(\frac{b Q}{b Q+(b-1) H(t-\tau)}\right) \\
& \Longleftrightarrow \frac{\mathrm{d} H(t)}{\mathrm{d} t}+\frac{(b-1) \mu}{b Q} H(t-\tau) \\
&=\frac{\mu((b-1) H(t-\tau))^{2}}{(b Q)^{2}+b Q(b-1) H(t-\tau)} \\
& \triangleq f(H(t), H(t-\tau)) .
\end{aligned}
$$

Figure 3(a) illustrates an example where the ERICA+ algorithm is not stable. The parameters considered in this example are the following: the available bandwidth is $\mu=100000$ cells/s, the threshold $Q$ is set to 200 cells and $\tau=0.064 \mathrm{~s}$. From Proposition 4.1, we get $\tau_{0}=0.06597$ for $b=1.05$, and $\tau_{0}=0.02408$ for $b=1.15$. Hence, we deduce that the instability region is between these two values. In many examples, we found that ERICA+ is unstable if $\tau>\tau_{0}$, where $\tau_{0}$ is the value obtained for $b=1.05$. Figure 3(a) confirms these claims.

With a slight modification of the ERICA+ algorithm, we can choose the parameters to ensure stability in all (a)

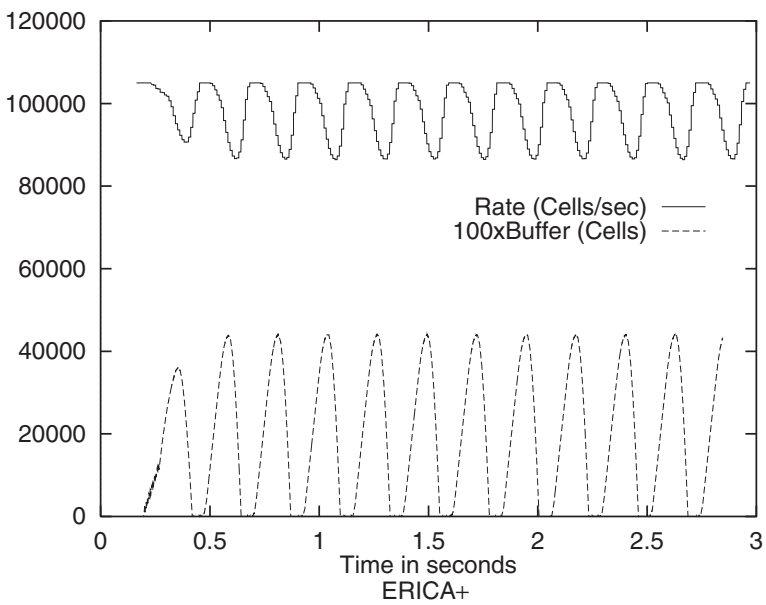

(b)

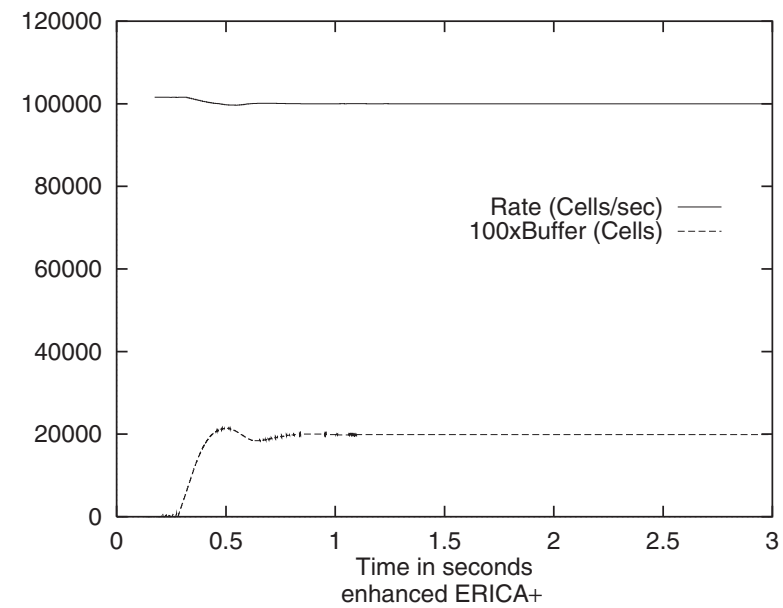

Figure 3. Buffer occupancy $(100 \times$ cells $)$ and the rate $($ cells $/ \mathrm{s})$, for $\tau=0.064 \mathrm{~s}, \mu=100000$ cells $/ \mathrm{s}$ and $Q=200$ cells for $E R I C A+$ (before and after introducing a new function $f n$ (Queue)).

cases. (The stability proof is as in Proposition 4.1). For example, instead of $f n($ Queue $) \triangleq b Q /((b-1) y(t)+Q)$, we define $f n$ (Queue) as:

$$
f n(\text { Queue }) \triangleq \frac{Q}{b y(t)+(1-b) Q},
$$

where

$$
b \triangleq\left\{\begin{array}{c}
\min \left(0.05, \frac{\pi Q}{6 * E R * \tau_{\max }}\right) \\
\text { if queue size }<Q \\
\min \left(0.15, \frac{\pi Q}{2 * \mathrm{ER} * \tau_{\max }}\right) \\
\text { otherwise }
\end{array} .\right.
$$

We call this version enhanced ERICA+. Figure 3(b) shows the effects of the new function $f n$ (Queue) and $b$. 
The rate as well as the queue length are constant rather than oscillatory (as it was the case in ERICA+).

\section{Conclusion}

In the present work, the stability of different ABR congestion control algorithms was studied, and the stability domain of each was stated. Although the Fixed Point algorithm was, fair and very simple, it guaranteed no stability under some configurations. The ERAQLES algorithm allowed a very fast convergence, however, the buffer size at intermediate switches could be very large (very aggressive). Finally, we showed that even for ERICA+ there were situations where the algorithm was not stable, and proposed values for parameter $b$, which enabled one to get better performances.

ERICA+ is known for the multiplicity of parameters. For the fairness purpose and full utilization, ERICA+ has to store the Last_Allocated_rate for each active connection, its contribution, the average active sources, CCR for each connection, and so on. This constitutes the main inconvenience of ERICA. We are currently working on a new explicit rate scheme that enables us to get better performance than ERICA+. This scheme only requires information on the CCR of each connection and the number of established ABR connections.

\section{References}

ATM Forum Technical Committee, 1996, Traffic Management Specification, Version 4.0, April 1996.

Ait-Hellal, O., and Altman, E., 1999, Performance evaluation of the rate-based flow control mechanism for ABR service: generalization. IEEE INFOCOM'99, New York, March, 1999, pp. 819-826.

Altman, E., BAŞAR, T., and SRIKANT, R., 1999, Congestion control as a stochastic control problem with action delays. In Control Issues in Telecommunication Networks, December 1999, pp. 1937-1950.

Beumohamed, L., and Meerkov, S. M., 1993, Feedback control of congestion in packet switching networks: the case of a single congested node. IEEE/ACM Transactions on Networking, 1, 693-708.

Bellman, R., and CoOKe, K. L., 1963, Differential-Difference Equations (New York: Academic press).

Brakmo, L. S., and Peterson, L. L. 1995, TCP Vegas: end to end congestion avoidance on a global internet. IEEE Journal on Selected Areas in Communications, 13, 1465-1480.

Brouillet, M. O., and Madhow, U., 1996, Rate control for adaptive bit rate sources on ATM networks using one bit congestion notification. Research Report UILU-ENG-96-2214, University of Illinois at Urbana-Champaign, May.

Chang, Y., Golmie, N., and Siu, D., 1995, A rate-based flow control switch designfor ABR service in an ATM network. Twelfth International Conference on Computer Communication ICCC'95, August 1995.

Charny, A., Clark, D. D., and Jain, R., 1995, Congestion control with explicit rate indication. Proceedings of ICC'95, June 1995.

Chong, S., Lee, S. and Kang, S., 2001, A simple, scalable, and stable explicit rate allocation algorithm for MAX-MIN flow control with minimum rate guarantee. IEEE/ACM Transactions on Networking, 9, 322-335.
Fulton, C. A., Li, S. Q., and Lim, C. S., 1997, An ABR feedback control scheme with tracking. IEEE INFOCOM'97, Kobe, Japan, April 1997, pp. 806-815.

Holma, H. and Toskala, A., 2001, WCDMA for UMTS, revd edn (Chichester: Wiley).

Hollot, C. V., Misra, V., Towsley, D., and Gong, W.-B., 2001, A control theoretic analysis of RED. Proceedings of IEEE, Infocom, Ankorage, Alaska, 2001.

Jain, R., Kalyanaraman, S., Goyal, R., Fahmy, S., and VISWANATHAN, R., 1996, ERICA switch algorithm: a complete description. ATM Forum/96-1172. [http:/www.cis.ohio-state.edu/ jain/atmf/a96-1172.htm.]

Jain, R., Kalyanaraman, S., and Viswanathan, R., 1995, The OSU scheme for congestion avoidance in ATM networks using explicit rate indication. Proceedings of the WATM'95 First Workshop on ATM Traffic Management, Paris, December 1995.

JAIN, R., 2003, Congestion control and traffic management in ATM networks: recent advances and a survey. Computer Networks and ISDN Systems, 28, 1723-1738

Jain, R., Kalyanaraman, S., Goyal, R., and Fahmy, S., 1996, Source behavior for ATM ABR traffic management: an explanation. IEEE Communications Magazine, November.

JOHARI, R., and TAN, D., 2001, End-to-end congestion control for the Internet: delays and stability. IEEE/ACM Transactions on Networking, 9, 818-832.

Khalil, H., 1996, Nonlinear Systems, 2nd edn (Upper Saddle River: Prentice Hall).

KIM, B. K., KIM, B. G., and ChONG, I., 1996, Fixed point algorithm for ABR congestion control. AF-TM 96-0061, April.

KuAng, Y., 1993, Delay Differential Equations, with Applications In population Dynamics (New York: Academic press).

KunNiYur, S., and SRIKANT, R., 2001, A time scale decomposition approach to adaptive ECN marking. IEEE INFOCOM'01, Anchorage, Alaska, April 2001.

MORET, Y. and FDIDA, S., 1997, ERAQLES an efficient explicit rate algorithm for ABR. GLOBECOM'97, Phoenix, AZ, USA, November 1997.

Massoulie, L., 2002, Stability of distributed congestion control with heterogeneous feedback delays (IEEE Transactions on Automatic Control, 47, 895-902) [available at http://research.microsoft.com/ users/lmassoul/].

Ohsaki, H., Hasegawa, G., Murata, M., and Miyahara, A., 1995a, Parameter tuning of rate-based congestion control algorithms and its application to TCP over ABR. In Proceedings WATM'95 First Workshop on ATM Traffic Management, Paris, December 1995.

Ohsaki, H., Murata, M., Suzuki, H., Ikeda, C., and Miyahara, H., 1995b, Rate-based control for ATM Networks. Computer Communication Review, ACM-Sigcomm, 25, 60-71.

Paganini, F., Doyle, J., and Low, S., 2001, Scalable laws for stable network congestion control. Proceedings of IEEE Conference on Decision \& Control, 2001.

RAMAKRISHNAN, K., and JAIN, R., 1988, A binary feedback scheme for congestion avoidance in computer networks with connectionless network layer. In Proceedings of SIGCOMM'88, August 1988.

RitTER, M., 1996, Network buffer requirements of the rate-based control mechanism for ABR services. IEEE INFOCOM'96, San Francisco, CA, USA, March 1996.

Shakkottai, S., Srikant, R., and Meyn, S. P., 2001, Boundedness of utility function based congestion controllers in the presence of delay. In Proceedings of IEEE Conference on Decision \& Control, 2001.

SiU, K. Y., and TzEnG, H. Y., 1995, Intelligent congestion control for ABR service in ATM networks. Computer Communication Review, ACM-Sigcomm, 24, 81-106.

VAN JACOBSON, 1998, Congestion avoidance and control. In $A C M$ SIGCOMM 88, pp. 273-288 [ftp.ee.lbl.gov/papers/congavoid.ps.Z].

VINNICOMBE, G., 2003. On the stability of networks operating TCPlike congestion control. In IFAC'02 (in press) [available at http:// www-control.eng.cam.ac.uk/gv/internet/].

Yin, N., and HluchyJ, M. G., 1994, On closed-loop rate control for ATM cell relay networks. In Proceedings of IEEE INFOCOM' '94, June 1994, pp. 99-108. 